\title{
Electron crystallography as a complement to X-ray powder diffraction techniques
}

\section{Review Article}

Author(s):

McCusker, Lynne B.; Bärlocher, Christian

Publication date:

2013

Permanent link:

https://doi.org/10.3929/ethz-b-000061049

Rights / license:

In Copyright - Non-Commercial Use Permitted

Originally published in:

Zeitschrift für Kristallographie 228(1), https://doi.org/10.1524/zkri.2013.1558 


\title{
Electron crystallography as a complement to $\mathrm{X}$-ray powder diffraction techniques
}

\author{
Lynne B. McCusker* and Christian Baerlocher \\ Laboratory of Crystallography, ETH Zurich, Switzerland \\ Dedicated to Professor Sven Hovmöller on the occasion of his 65th birthday
}

Received July 10, 2012; accepted September 19, 2012

Published online: October 22, 2012

$X$-ray powder diffraction / HRTEM / SAED / PED structure solution

\begin{abstract}
Electron microscopy techniques yield information for crystal structure analysis that is remarkably complementary to that obtained from X-ray powder diffraction data. Structures of polycrystalline materials that resist solution by either method alone can sometimes be solved by combining the two. For example, the intensities extracted from an X-ray powder diffraction pattern are kinematical and can be interpreted easily, while those obtained from a typical selected area electron diffraction (SAED) or precession electron diffraction (PED) pattern are at least partially dynamical and therefore more difficult to use directly. On the other hand, many reflections in a powder diffraction pattern overlap and only the sum of their intensities can be measured, while those in an electron diffraction pattern are from a single crystal and therefore well separated in space. Although the intensities obtained from either SAED or PED data are less reliable than those obtained with Xrays, they can be used to advantage to improve the initial partitioning of the intensities of overlapping reflections. However, it is the partial crystallographic phase information that can be extracted either from high-resolution transmission electron microscopy (HRTEM) images or from PED data that has proven to be particularly useful in combination with high-resolution X-ray powder diffraction data. The dual-space (reciprocal and real space) structure determination programs Focus and Superflip have been shown to be especially useful for combining the two different types of data.
\end{abstract}

\section{Introduction}

X-ray powder diffraction and electron microscopy are probably the two most valuable techniques for analyzing the structures of polycrystalline materials. Although it is relatively easy to measure an X-ray powder diffraction pattern, a detailed interpretation of the data is not always straightforward. Reflections from differently oriented crystallites are

\footnotetext{
* Correspondence author (e-mail: lynne.mccusker@mat.ethz.ch)
}

measured simultaneously, so reflections that happen to have similar $d$-spacings (and therefore similar diffraction angles) overlap in the powder pattern. This hinders structure elucidation by conventional (single-crystal) crystallographic methods, because the algorithms assume that the intensities are reliable. However, a wide variety of approaches have been developed to deal with this overlap problem, and now structures of moderate complexity can be solved from powder diffraction data in an almost routine manner [1].

Because electrons interact with matter much more strongly than do X-rays, very tiny crystals $(<100 \mathrm{~nm})$ can be examined individually in an electron microscope. Furthermore, because electrons can be focussed, both diffraction patterns and high-resolution images can be obtained. Unfortunately, the operation of an electron microscope requires considerable expertise, whether it is to collect a complete set of good electron diffraction data or to record a high-resolution image. Until quite recently, electron diffraction data collection was limited to sets of isolated 2-dimensional patterns that had to be merged with one another to form an incomplete set of 3-dimensional data. Efforts to devise a more automated and complete data collection strategy are now quite advanced [2, 3], so this limitation can be expected to be reduced in future. Although electron diffraction intensities are distorted by a number of effects that are not easy to control or correct for, electron crystallography has been applied successfully to various classes of materials $[4,5]$.

Table 1. Comparison of X-ray Powder and Electron Diffraction.

\begin{tabular}{lll}
\hline & X-ray Powder & Electron \\
\hline Data collection & easy & difficult \\
Data set & complete & incomplete \\
Lattice parameters & precise & approximate \\
Intensities & kinematic & dynamical \\
Multiple diffraction & no & yes \\
Systematic absences & difficult & easy \\
Crystallite size & $\mu \mathrm{m}$ & nm \\
Reflection overlap & yes & no \\
Phase information & no & yes \\
\hline
\end{tabular}


These two techniques are remarkably complementary (see Table 1), and several ways of combining them have been developed to address structures that cannot be solved by either method alone. In the following sections, the relevant features of the two techniques, the algorithms used to combine the different kinds of data, and some recent examples are described.

\section{X-ray powder diffraction}

\subsection{The overlap problem}

The main limitation of X-ray powder diffraction data is reflection overlap. In most cases, the total intensity measured for a group of overlapping reflections is simply equipartitioned over the contributing reflections. The problem can be reduced by collecting high-resolution data at a synchrotron facility, but it cannot be eliminated entirely. Several approaches that use the intensities of the non-overlapping reflections to estimate those of the overlapping ones have been developed $[6,7,8]$, but for a diffraction pattern with a high degree of overlap these methods are not very reliable. In such cases, more elaborate data collection strategies, in which several diffraction patterns are measured on the same sample under different conditions, can be employed (e.g. [9-11]). All of the measured patterns are then used simultaneously to derive a single set of reflection intensities, which are more single-crystal-like in character than those obtained in a conventional manner. Whichever approach is used, however, a certain degree of ambiguity in the intensities of the overlapping reflections remains and must be taken into consideration during structure solution.

\subsection{Structure determination from powder diffraction data}

Crystallographers have developed and optimized a number of methods to address the phase problem for single-crystal data (e.g. [12]), and direct methods programs are now used to solve the vast majority of structures for which single crystals are available. However, these algorithms assume that the reflection intensities are reliable, because relationships between sets of reflections and their intensities are used to estimate the phases. With powder diffraction data, of course, this condition is not fulfilled. When it became apparent in the late 1980's that structure solution from powder diffraction data was indeed feasible given the improved instrumentation and increasing computer power, the existing methods were adapted where possible to deal with the reflection overlap problem (e.g. EXPO [13], XLENS [14] and MICE [15]).

However, it was soon recognized that alternative approaches would be required for diffraction patterns with severe overlap. Supplementing the powder diffraction data with chemical information (known bond distances and angles, coordination numbers, connectivity, etc.) was found to be a particularly effective approach [1]. This resulted in a number of automated model-building programs based on global optimization algorithms (both evolutionary and si- mulated annealing) that used the powder diffraction pattern to identify the better models during the course of the optimization. By working in real or model-building (direct) space, these algorithms bypass the problem of reflection overlap, because the intensities of the individual reflections are not needed. Only the diffraction pattern as a whole is used to assess the reliability of the models generated during the structure solution procedure. For more detailed information, the reader is referred to the book Structure Determination from Powder Diffraction Data [16] and the special issue of $Z$. Kristallogr. devoted to the same topic [11].

While global optimization algorithms work in direct space, direct methods operate in reciprocal space (i.e. with reflection intensities and phases). A few algorithms, such as the shake-and-bake modification to direct methods [17], the charge-flipping algorithm that was introduced only recently [18], and the zeolite-specific program Focus [19], alternate between the two spaces and can therefore benefit from the individual strengths of both.

\subsection{Structure envelopes}

It is possible to facilitate structure determination in direct space by identifying the regions within the unit cell that are most likely to contain atoms. For this purpose, the concept of a structure envelope was introduced [20]. The main difference between this structure envelope and the molecular envelope that is used in protein crystallography is that the former does not necessarily have a closed form. It is a periodic nodal surface [21] that separates regions of high electron density from those of low electron density, and can be generated from just a few strong, low-index reflections [22].

In the case of a zeolite, this curved surface describes the pore system (e.g. Fig. 1), with the framework atoms lying on the positive side of the surface. For a molecular structure it is a closed envelope that describes the approximate positions, orientations and shapes of the molecules in the unit cell.

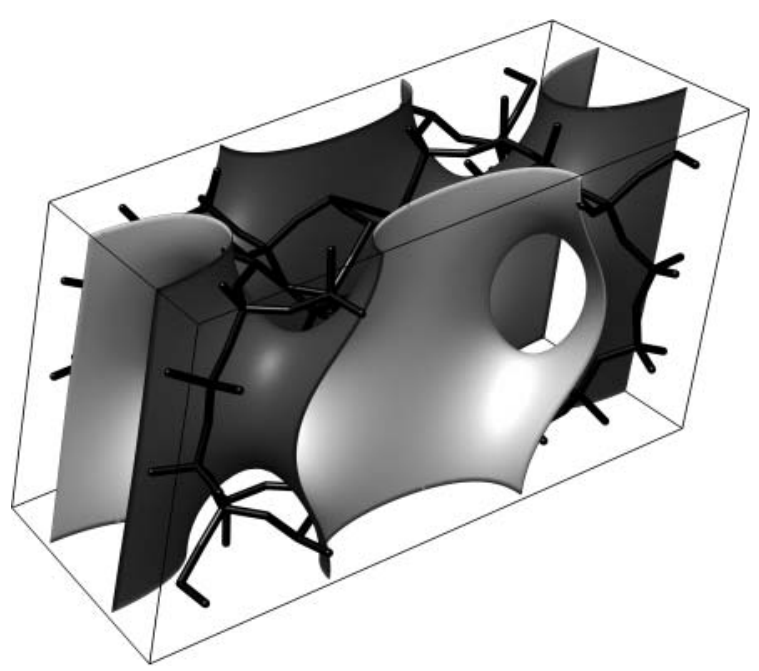

Fig. 1. The framework structure of the zeolite CIT-1 with a structure envelope generated using just four strong, low-index reflections. Note that the framework atoms all lie on the dark gray (positive) side of the structure envelope. 


\section{Electron microscopy}

Although electron crystallography has a long history [4], it has never been a mainstream technique for crystal structure determination. The reason is that the diffraction intensities are strongly affected by dynamical/multiple scattering. Because the electrons interact with the sample so strongly (ca. $10^{3}$ times more than X-rays), the diffracted beams are also strong and can, in turn act as incident beams. Thus, the interaction between the incident beam and the crystal cannot be treated as a single-scattering event for each reflection as it is for X-rays (kinematical approximation). The system must be treated as a whole to account for multiple scattering. That is, all simultaneously excited reflections must be considered together. The thicker the sample, the more severe this effect.

\subsection{Selected area electron diffraction (SAED)}

Dynamical effects can be minimized by selecting a very thin crystal ( $\sim 50 \mathrm{~nm}$ or less). Then the diffraction intensities measured using a conventional selected area electron diffraction (SAED) technique can be treated as quasi-kinematical. For an SAED pattern, a very small area of the crystal is selected using an aperture below the sample holder. This approximation has been used successfully for structure analysis by a number of specialists $($ e.g. $[4,5]$ and references therein).

The $100-300 \mathrm{keV}$ acceleration voltages typically used in electron microscopes correspond to wavelengths of 0.037-0.020 ̊. This means that the Ewald sphere has a very large radius $(1 / \lambda)$, so many reflections fulfill the diffraction condition at once (Fig. 2). In a typical SAED pattern, the incident beam is generally aligned along a zone axis, and the complete zero-order diffraction pattern for that zone (zero order Laue zone or ZOLZ) can be recorded out to a resolution of less than $1 \AA$ in a single shot. However, because many reflections are excited simultaneously, multiple scattering effects can be severe, particularly if the specimen is not ideally thin.

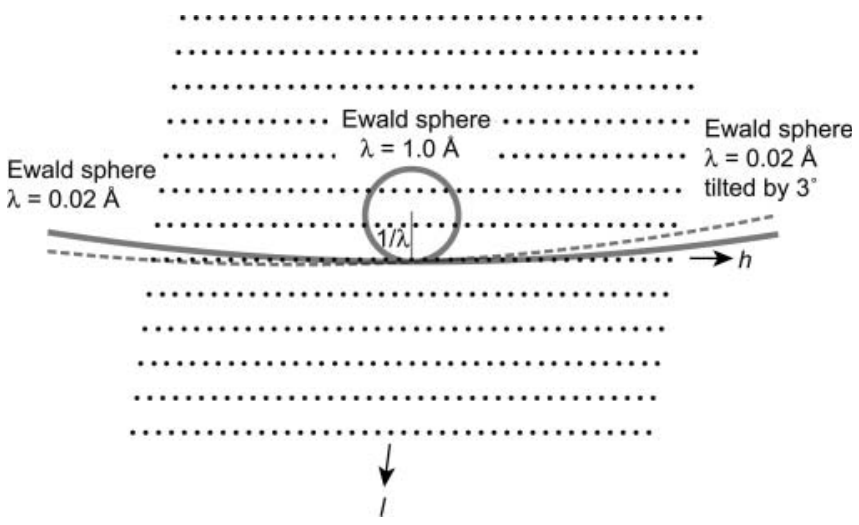

Fig. 2. Ewald construction for a $1.0 \AA$ (typical X-ray) and for a $0.02 \AA$ (typical EM) wavelength. The effect of tilting the EM Ewald sphere by $3^{\circ}$ (typical precession angle) is also shown (dashed line). Note that the intersection of the Ewald sphere $($ diameter $=1 / \lambda)$ with points in reciprocal space (i.e. reflections in diffracting condition) is much larger with the smaller wavelength and that this number is reduced by tilting.

\subsection{Precession electron diffraction (PED)}

The precession electron diffraction (PED) technique, which reduces the dynamical scattering [23], was first introduced by Vincent and Midgley in 1994. The electron beam is deflected and then precessed around a stationary crystal, and with this tilted illumination, only a few reflections are excited at a time, so the possibilities for multiple scattering are reduced and the resulting diffraction intensities are more kinematical in nature (Fig. 2). Although the PED intensities are still not ideal, they are significantly improved over typical SAED intensities [24], and have been used to solve several structures [2529].

\subsection{High-resolution transmission electron microscopy (HRTEM)}

Because the electron beam can be focussed, it is also possible to record a magnified image of the sample with an electron microscope. The magnification attainable depends upon the wavelength, and resolution in the subnanometer range is possible.

The advantages of having a high-resolution transmission electron microscope (HRTEM) image for structure analysis are twofold. First, the image shows approximately where the atoms are in that projection of the unit cell even if atomic resolution is not attained, so the validity of a structure envelope can be evaluated by eye. Second, the Fourier transform of the image yields a list of structure factor amplitudes and phases for the reflections in the corresponding diffraction pattern.

Before the Fourier transform is performed, a contrasttransfer-function (CTF) correction, which allows for the modulation of the amplitudes and phases caused by various parameters of the microscope (spherical aberration of the objective lens, wavelength, defocus and spatial frequency), is applied. Then the image is lattice and symmetry averaged to obtain a clearer and defect-free image. Fourier transform of this modified image then generates the desired phase information. The crystallographic image processing software package CRISP [30] can be used to perform these calculations.

It has been found that including even the limited phase information that can be derived from a single projection can have significant impact on structure solution from powder diffraction data [31].

\section{Dual-space structure solution algorithms}

It was mentioned earlier that a few structure determination algorithms work in both reciprocal and direct space, so information from different sources can be added in either realm quite easily. Such algorithms are particularly well-suited for combining data from different sources. In the last few years, two of them, Focus and powder charge flipping $(p \mathrm{CF})$, have been used successfully to combine X-ray powder diffraction and electron microscopy data. 


\subsection{Focus}

Focus is a zeolite-specific structure-solution program that uses chemical information (direct space) to compensate for the ambiguities in the reflection intensities (reciprocal space) extracted from a powder diffraction pattern [19]. Structure factor amplitudes are calculated from the extracted intensities (overlapping reflections are usually equipartitioned), and are then assigned starting phases (usually random). These are used to generate a (random) electron density map. The program then employs one of two methods to interpret the map and calculate a (partial) model. It is primarily in this interpretation step that the chemical information about zeolite framework structures (4-connected, 3-dimensional network of well defined corner-sharing $\mathrm{TO}_{4}$ tetrahedra, where $T$ is any tetrahedrally coordinated atom such as $\mathrm{Si}$ or $\mathrm{Al}$ ) is exploited. New phases are then calculated from the structural model and applied to the extracted intensities to generate a new electron density map and this Fourier recycling procedure continues until the phases converge or a maximum number of cycles is reached. Each time an electron density map is generated, the program conducts an exhaustive search for a 3-dimensional, 4-connected framework structure. If one is found, it is classified and written to a file. When a sufficient number of frameworks have been found, a histogram is generated, and in general, the framework found most frequently (from different starting phase sets) will be the correct one. The algorithm has now been applied by many different laboratories to data collected on a wide variety of zeolites with considerable success. Its only drawback is that it is specific to zeolites.

In 2005 the germanosilicate ITQ-22 [32] had the most complex zeolite framework structure known (16 Si/Ge atoms in the asymmetric unit), so it was used to test the value of including some initial phases in the Focus input [31]. These tests showed that by prescribing the phases of just 31 of the 992 strongest reflections, the time required for structure solution could be reduced from 31 days to 44 hours. Doubling the number of phases to 62 resulted in 17 solutions within 11 hours. Obviously even a limited amount of phase information has a significant effect on the structure solution process.

\subsection{Powder charge flipping}

In 2004, Oszlányi and Sütő introduced a more general algorithm for structure solution from single-crystal data (Fig. 3), that they called charge flipping [33, 34]. It starts in much the same way as does Focus. That is, random phases are assigned to the set of structure factor amplitudes $\left(\left|F_{h k l}\right|\right)$ derived from the diffraction intensities, and an electron density map $(\varrho(r))$ is generated. Then the signs of all electron density points below some user-defined threshold $\delta$ (a small positive number) are reversed ("flipped") to produce a perturbed electron density map $(g(r))$. In effect, all negative electron densities, which are not physically meaningful, are made positive. From this map, a new set of structure factor amplitudes $\left(\left|G_{h k l}\right|\right)$ and phases $\left(\varphi_{h k l}\right)$ are calculated, and the new phases are combined with the measured amplitudes $\left(\left|F_{h k l}\right|\right)$ and a new

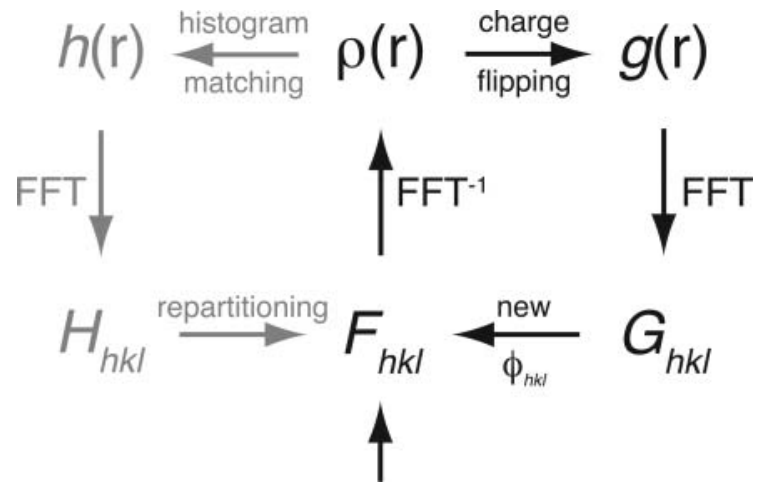

Fig. 3. A simple outline of the charge flipping algorithm. The original single-crystal cycle is shown in black, and the loop for repartitioning the intensities of overlapping reflections that was added for powder diffraction data is shown on the left in gray. The latter is executed at periodic intervals during the course of a run.

electron density map is calculated. This cycle is repeated until the calculated structure factor amplitudes match the measured ones or until a preset number of cycles has been reached.

To accommodate powder diffraction data, something had to be done about the relative intensities of overlapping reflections. In the implementation by $\mathrm{Wu}$ et al. [35], the ratios of the calculated structure factor amplitudes $\left(\left|G_{h k l}\right|\right)$ in each overlap group are used to repartition the measured intensity. In the $p C F$ algorithm by Baerlocher, McCusker and Palatinus [36], which is implemented in the program Superflip [37], an additional modification to the map based on a histogram-matching algorithm [38] is performed $(h(r))$, and then the structure factor amplitudes $\left|H_{h k l}\right|$ calculated from this map are used for the repartitioning step. This second type of perturbation of the electron density map simply forces it to reflect the chemical composition of the material under investigation, and has proven to be quite effective. Histogram matching has a beneficial side effect in that it also eliminates the "uranium atom" solution trap that the charge flipping algorithm is prone to.

\section{Combining X-ray powder diffraction data with HRTEM images}

Data from high-resolution electron microscopy images can be supplied to the Focus and $p \mathrm{CF}$ algorithms in several different ways. For example, a structure envelope for a zeolite derived from one or more HRTEM images can be imposed in real space to limit the peak search in Focus or to set the electron density in the pore regions to zero in $p C F$. Alternatively, the phases of the reflections used to generate the structure envelope can be fixed in reciprocal space. It is also possible to include all phases obtained from HRTEM images (i.e. not just the few used to generate a structure envelope) in the starting phase set in either program. Although it would be possible to keep these phases fixed, experience has shown that it is more prudent to allow them to change during the normal procedure, whether in Focus or $p \mathrm{CF}$, to allow any incorrect phases to correct themselves. However, in $p \mathrm{CF}$, it is critical that they 
be fixed for the initial 10-20\% of the cycles if they are to be effective. A further option is to use an approximate or partial model that has been deduced from HRTEM images as a seed for generating starting phase sets.

Different combinations of these options eventually led to the determination of the three most complex zeolite structures known (TNU-9 [39], IM-5 [40] and SSZ-74 [41]). The relevant aspects of these structure solutions are given in the following sections to illustrate the procedure. More information can be found in the original papers and in the Chem. Commun. review article by McCusker and Baerlocher [42]. Although all three materials are zeolites, only the first was solved using the zeolite-specific program Focus. The more generally applicable $p \mathrm{CF}$ algorithm was used for the other two, and the concepts applied in all three cases should be readily transferable to non-zeolite materials.

\subsection{TNU-9}

The first true test of the value of including phase information in the Focus algorithm was performed on the highsilica zeolite TNU-9 [43], whose structure was unknown. Most of the peaks in the high-resolution synchrotron powder diffraction pattern could be indexed with a monoclinic unit cell $(C 2 / m, a=28.2219 \AA, b=20.0123 \AA$, $c=19.4926 \AA, \beta=92.33$ ), but a few smaller peaks remained unindexed. Therefore, SAED data were used to verify that these were indeed impurity peaks and to confirm the unit cell of the main phase. Attempts to solve the structure with Focus using the powder diffraction data alone, however, were not successful.

From three high-quality HRTEM images of TNU-9 (along the [010], [001] and [110] zone axes), the phases of 258 reflections were estimated using the CRISP software package. As an example, the image taken along the [010] zone axis is shown in Fig. 4. Only when all of these phases were included in the Focus input, could the structure, with $24 \mathrm{Si}$ and $52 \mathrm{O}$ atoms in the asymmetric unit, be solved. Structure solution required 16 days of CPU time.

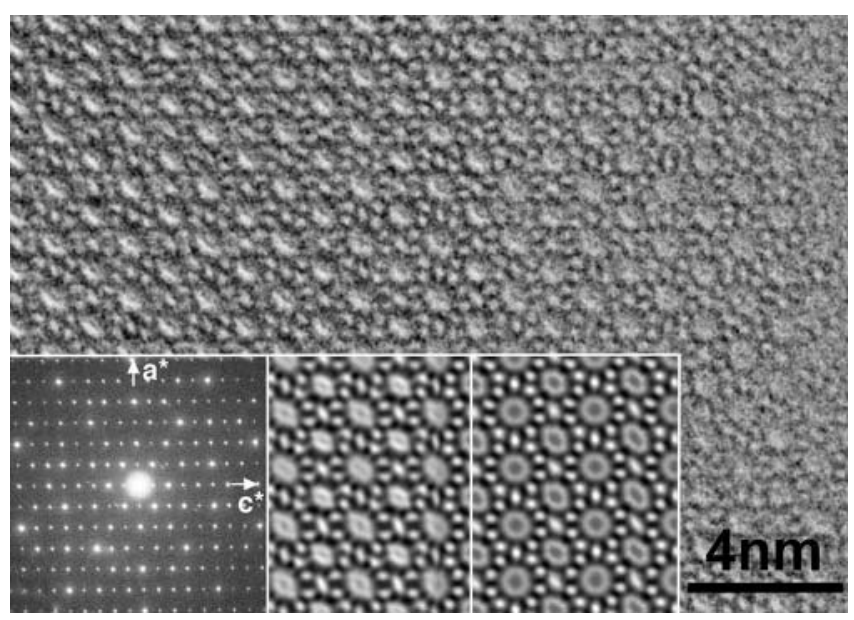

Fig. 4. HRTEM image of TNU-9 taken along the [010] zone axis [39]. The insets show the corresponding SAED pattern (left), the contrast-transfer-function corrected and symmetry-averaged image, from which the phases were calculated (middle), and the computer simulation from the structural data (right).

\subsection{IM-5}

In the case of IM-5 [44], another high-silica zeolite, a slightly different approach was used. As for TNU-9, impurities were present, so SAED experiments were performed to verify the unit cell ( $\mathrm{Cmcm}, a=14.299 \AA$, $b=57.413 \AA$ and $c=20.143 \AA$ ).

Initially, the phases of 95 reflections were estimated from three HRTEM images (along the [100], [010] and [001] zone axes) and included in $p$ CF runs, but the resulting electron density maps could not be interpreted on an atomic level. After careful corrections to the HRTEM images, a 3-dimensional potential map was generated in the space group $C 2 \mathrm{~cm}$ using just 71 phases (Fig. 5) and a model with $36 \mathrm{Si}$ atoms in the asymmetric unit deduced. Although this structural model was geometrically strained and its calculated powder pattern did not fit the measured one very well, it was reasoned that it was probably at least partially correct (certainly more so than a random structure). Therefore it was used as a seed to generate 1000 different starting phase sets for $1000 p \mathrm{CF}$ runs. That is, the phase of each structure factor calculated from the geometrically optimized model was varied by up to $25 \%$ in a random manner. The 1000 electron density maps resulting from these $p \mathrm{CF}$ runs were symmetry averaged assuming the space group $C 2 \mathrm{~cm}$, and then the best five were combined. Interpretation of this map was almost trivial: 35 of the $36 \mathrm{Si}$ atoms and 61 of the $79 \mathrm{O}$ atoms could be located directly in the strongest 106 peaks (Fig. 6). The position of the missing $\mathrm{Si}$ atom was derived from the positions of the four surrounding $\mathrm{Si}$ atoms and the missing bridging $\mathrm{O}$ atoms were added. Rietveld refinement of this model revealed that additional symmetry was present in the structure. That is, atomic positions related by a center of symmetry (not present in $\mathrm{C} 2 \mathrm{~cm}$ ) were found to be insignificantly different from one another, so the structure
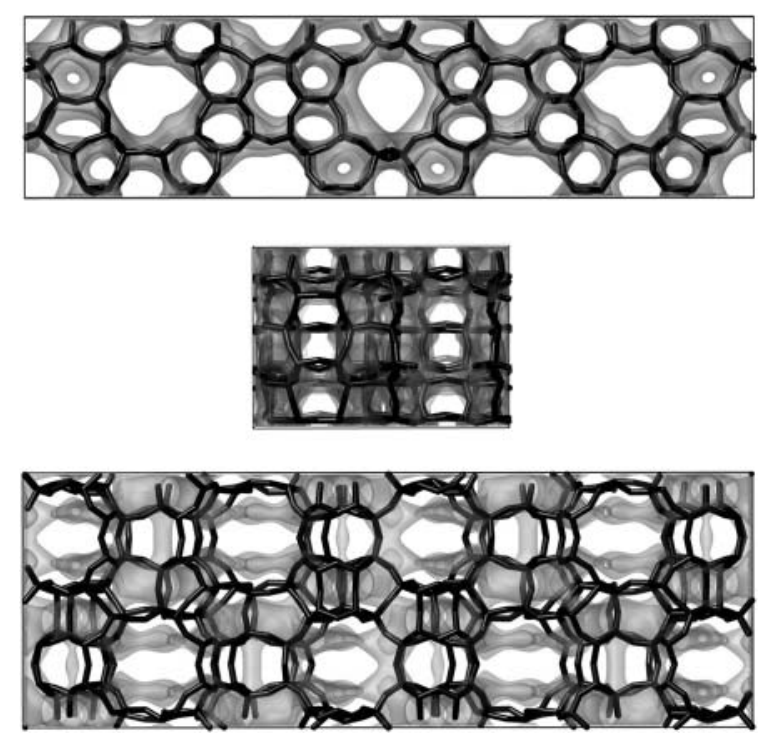

Fig. 5. Potential maps for the [001], [010] and [100] projections (top to bottom) of IM-5 generated in $C 2 \mathrm{~cm}$ from the 71 amplitudes and phases estimated from the HRTEM images. It can be seen from the refined framework structure that has been superimposed, that a considerable amount of structural information is contained in these few reflections. However, some of the finer details, particularly in the [100] projection, are incorrect. 


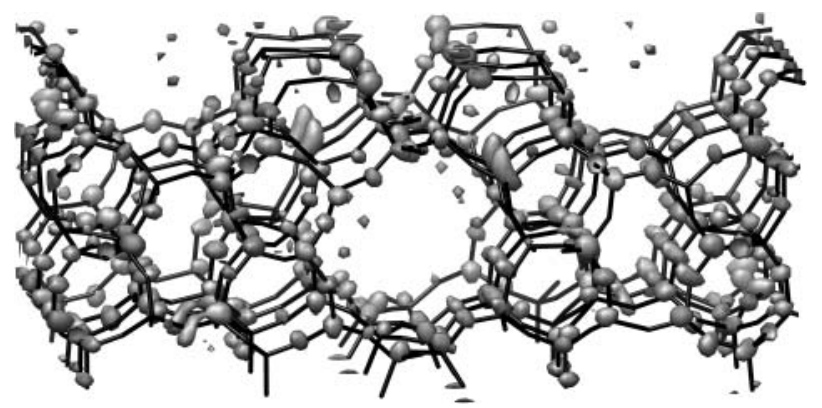

Fig. 6. Electron density map from the $p \mathrm{CF}$ algorithm in Superflip that was used to derive the structure of IM-5. To allow more detail to be seen, only a portion of the unit cell is shown. The refined framework structure has been superimposed for comparison.

could be described in the higher space group $\mathrm{Cmcm}$ with $24 \mathrm{Si}$ atoms in the asymmetric unit.

Improved HRTEM images of IM-5 were later used to test the limits of electron crystallography [45]. From the systematic absences in the SAED patterns and the projections symmetries of the HRTEM images, the correct space group $\mathrm{Cmcm}$ was obtained. It was possible to derive 144 structure factor amplitudes and phases from the set of HRTEM images, and these produced a potential map from which the positions of all $24 \mathrm{Si}$ atoms could be determined.

\subsection{SSZ-74}

The $p \mathrm{CF}$ approach was also applied to synchrotron powder diffraction data collected on the high-silica zeolite SSZ-74 [46]. In this case, no impurities were present and the pattern could be indexed with confidence with a monoclinic unit cell $(C 2 / c$ or $C c, a=20.514 \AA, b=13.395 \AA$, $\left.c=20.097 \AA, \beta=102.2^{\circ}\right)$. However, only one HRTEM image (taken along the [110] zone axis) of relatively low resolution was available, and only 29 structure factor amplitudes and phases could be derived from it. These were used to construct a structure envelope that could be imposed in real space in the $p \mathrm{CF}$ algorithm to eliminate any electron density in the pores (Fig. 7).

The best electron density maps generated from a series of $p \mathrm{CF}$ runs were examined by eye. Those judged to have realistic pores and at least a partial framework structure were averaged (Fig. 8b), and then used as a seed within
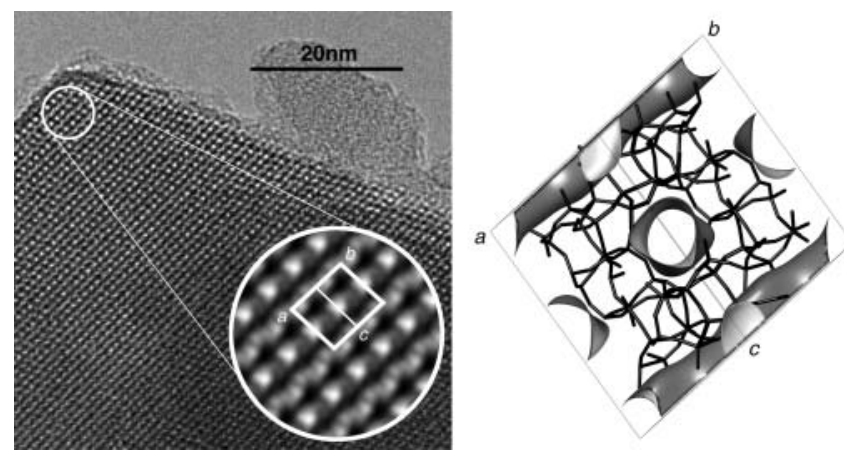

Fig. 7. The HRTEM image of SSZ-74 taken along the [110] direction (left) with an enlarged inset showing a translationally averaged image, and the structure envelope (right) calculated from the 29 structure factors derived from the image shown in the inset. The refined framework structure has been overlaid for comparison.
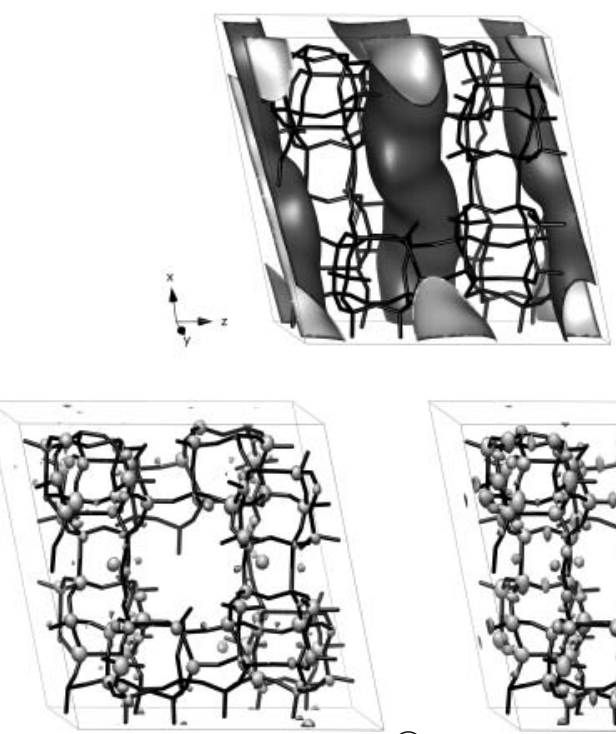

(b)

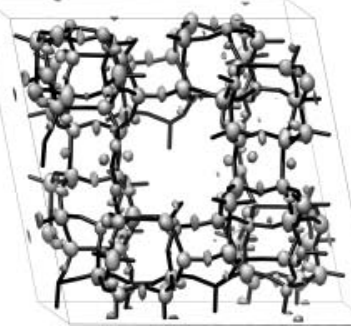

Fig. 8. Solution of the structure of SSZ-74. (a) The structure envelope (same as that shown in Fig. 7 but rotated), (b) The powder charge-flipping electron density map generated using random starting phases and the structure envelope. (c) The electron density map generated using the map shown in (b) as a seed. The electron density maps shown in both (b) and (c) are averages of the better solutions. The same electron density contour level was used for both maps.

the Superflip program to generate 100 new starting phase sets in a manner analogous to that used for IM-5 (but with a map in this case rather than a model). The ten best maps from these runs were then averaged (Fig. 8c), and the framework structure could be recognized easily.

The symmetry of this map was somewhat ambiguous, so the centrosymmetric space group $C 2 / c$ was assumed. Eleven Si positions were taken from the $p \mathrm{CF}$ map, and a twelfth one was added by hand to yield a fully 4-connected framework with a pattern very similar to the measured one. Subsequent Rietveld refinement showed that this twelfth Si position was in fact only half occupied, and that the twofold axis was not present. The space group symmetry was reduced to $C c$, and the final structure, with $23 \mathrm{Si}$ atoms in the asymmetric unit, proved to be a most unusual one with ordered Si vacancies.

\section{Combining X-ray powder with precession electron diffraction data}

Although it is readily apparent that HRTEM images can be extremely useful in the structure determination process, they are not always easy to obtain, especially if the sample is beam sensitive. To circumvent this problem, Xie et al. [47] investigated the possibility of using the less demanding precession electron diffraction (PED) technique in place of HRTEM. PED and X-ray powder diffraction data were used in combination by Dorset [48] to solve and refine the structure of the zeolite ZSM-10. However, in that case, the structure was solved from the PED data and then refined with the X-ray data. The question here is whether or not the two sets of data can be combined to facilitate structure solution. The PED data can be used in two ways: (1) to identify weak reflections to improve the 


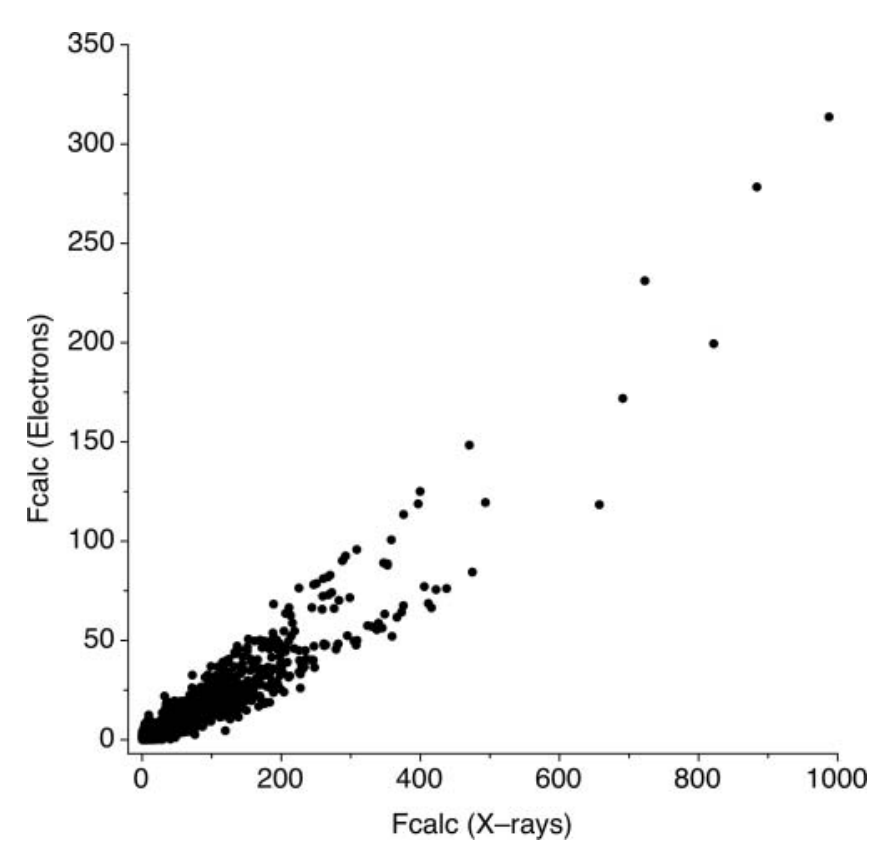

Fig. 9. Comparison of the structure factor amplitudes calculated for ZSM-5 with electron (vertical axis) and X-ray (horizontal axis) scattering factors. Although the correlation is not $100 \%$, it can be seen that the structure factor amplitudes that are weak in the electron diffraction case are also weak with X-rays.

powder diffraction intensity extraction, and (2) to estimate the phases of the reflections in the projection. Both strategies proved to have a significant and beneficial impact on the structure solution process. To keep things simple, the structure factors derived from the PED patterns were calculated assuming kinematical scattering and no geometric corrections were applied.

\subsection{Weak reflection elimination}

Although the scattering factors for X-rays and electrons are not identical, they do show the same general trend, so reflections that are weak in the electron diffraction pattern should also be weak in the corresponding X-ray diffraction pattern (Fig. 9) [49]. Thus, if those weak reflections are eliminated during the X-ray intensity extraction process, the intensities of the remaining reflections in an overlap group should be better estimated (Fig. 10).

Tests on the zeolite ZSM-5 (Pnma; $a=20.022 \AA$, $b=19.899 \AA, c=13.383 \AA$ [50]) using PED patterns from just four projections $([010],[01 \overline{2}],[02 \overline{1}]$ and $[10 \overline{1}]$ with a total of 594 reflections) showed that the electron density map generated by $p \mathrm{CF}$ with intensities derived using this weak reflection elimination (WRE) procedure improved significantly. Of the 3042 reflections in the pow-

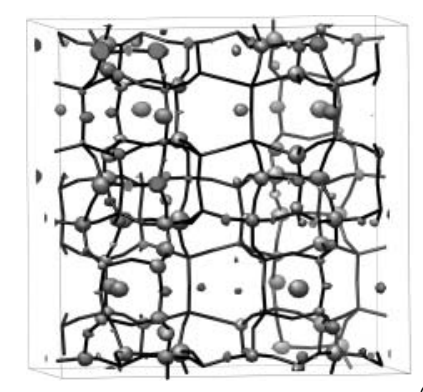

(a)

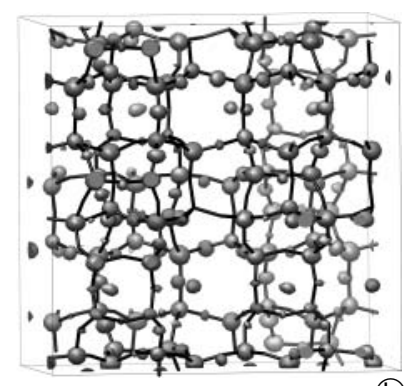

(b)

Fig. 11. Electron density maps (approximately along [001]) produced by $p \mathrm{CF}$ for ZSM-5 using (a) a normal intensity extraction procedure (all 3042 reflections, $R_{\text {map }} \sim 55 \%$ ), and (b) intensity extraction after elimination of 412 weak reflections from the reflection list (2630 reflections, $R_{\text {map }} \sim 25 \%$ ). The framework structure has been superimposed for comparison.

der diffraction pattern, 412 were defined to be weak and eliminated from the intensity extraction procedure. With the new set of intensities, the best maps produced by the powder charge flipping algorithm matched the correct one much better (Fig. 11). The agreement factor $R_{\text {map }}$, which compares the $p \mathrm{CF}$ map with a reference map calculated from the true structure, decreased from over $55 \%$ without WRE to less than $25 \%$ with WRE.

\subsection{Phase retrieval from PED data using charge flipping}

Precession electron diffraction data have already been used in combination with direct methods and maximum entropy methods to determine two-dimensional structures [25-28]. This is an indication that the reflection intensities are reliable enough and have sufficient resolution for the phasing process to succeed. For three-dimensional structures, of course, a single projection is insufficient to solve the structure, but the phases for selected projections can be used in combination with $\mathrm{X}$-ray powder diffraction data in the same way as those derived from 2-dimensional electron microscopy images.

Xie et al. used the single-crystal charge-flipping algorithm of Oszlányi and Sütô [18, 33, 34] implemented in the program Superflip [37] rather than direct or maximum entropy methods to estimate the phases. Using the same PED patterns for ZSM-5 as for the weak reflection elimination study, 100 charge-flipping runs, each with 500 iterations, were performed on each of the four twodimensional datasets (289 reflections for [010], 162 for $[01 \overline{2}], 124$ for $[02 \overline{1}]$ and 246 for $[10 \overline{1}])$. The resolution for each of these datasets was ca. $0.8 \AA$.
Fig. 10. The effect of weak reflection elimination on intensity extraction. The true intensities of three reflections $(10,7,1)$ under a single peak are shown in the center. On the left is a conventional equipartitioning $(6,6,6)$, and on the right, equipartitioning after the weak reflection has been eliminated $(9,9,0)$.
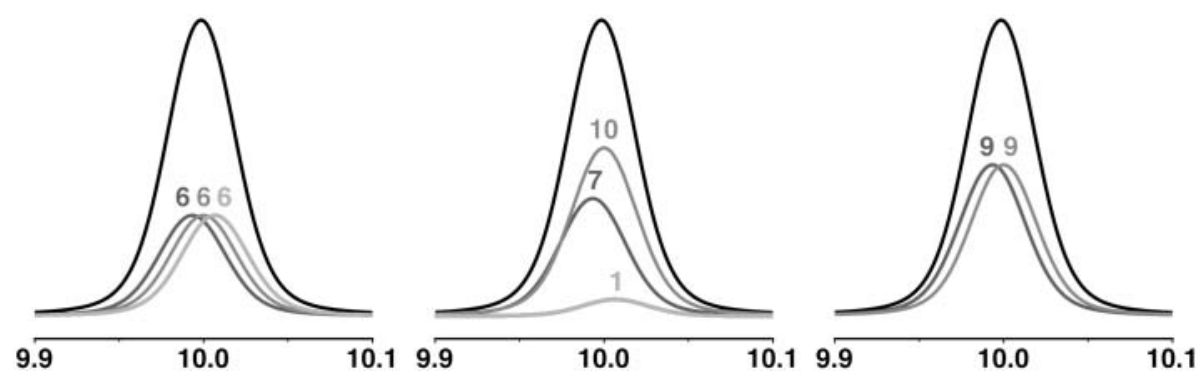
For each projection, the five maps with the best Superflip $R$-values were averaged. Then a Fourier transform was applied to calculate the phases of the corresponding reflections. Even though the symmetry of the map at this stage was $P 1$, the space group symmetry was imposed for this transformation to ensure that only phases consistent with a centrosymmetric space group were obtained. The total amplitude that was correctly phased was more than $70 \%$ for each of the four zones. This number compares well with that obtained for the phases derived from HRTEM images. Inclusion of the 594 phases from the four projections in the starting phase sets of $p \mathrm{CF}$ runs using the 3042 reflection intensities extracted from X-ray powder diffraction data led to a significant improvement in the resulting electron density maps ( $R_{\text {map }}$ reduced from $57 \%$ to $18 \%$ ).

\subsection{Combining weak reflection elimination and phase retrieval}

The combination of weak reflection elimination and phase retrieval was tested with PED data recorded for the zeolite TNU-9. Five PED patterns were used to eliminate 323 reflections and to derive 176 phases, and then $100 \mathrm{pCF}$ runs of 600 iterations each were performed. The best map showed the positions of 23 of the $24 \mathrm{Si}$ atoms in the asymmetric unit and many of the $\mathrm{O}$ atoms. However, the heights of the peaks did not reflect the relative scattering powers of $\mathrm{Si}$ and $\mathrm{O}$. Therefore, this map was used as a seed in Superflip to generate 100 new starting phase sets by allowing the phases calculated from the seed map to vary by up to $20 \%$ in a random fashion. The best electron density maps resulting from this second series of $p C F$ runs showed all $24 \mathrm{Si}$ atom positions and more realistic electron densities for Si and O (Fig. 12).

\section{Combining X-ray powder with selected area electron diffraction data}

Selected area electron diffraction patterns can also be used to advantage in conjunction with X-ray powder diffraction. Not only can the unit cell dimensions be verified, but the

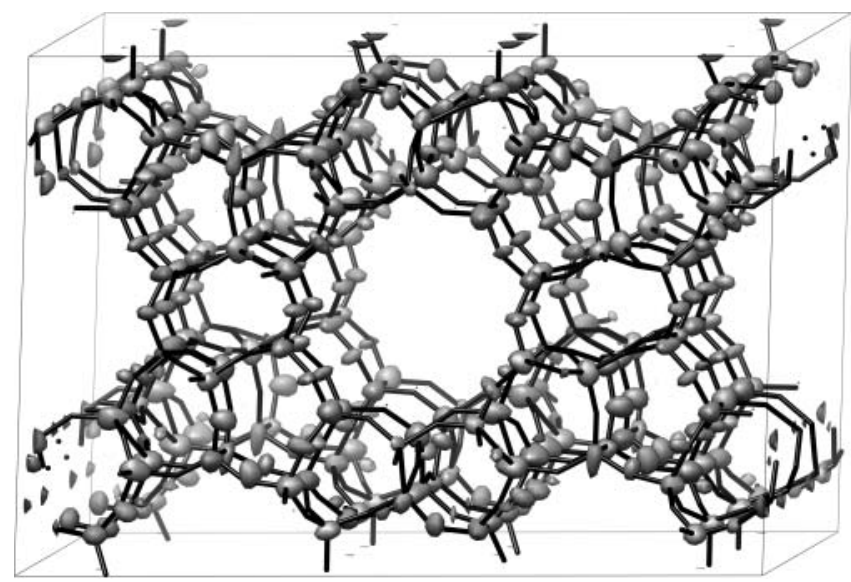

Fig. 12. Final powder charge flipping electron density map for TNU9 (approximately along [010]) obtained by combining weak reflection elimination and phase retrieval. The final framework structure is superimposed for comparison.

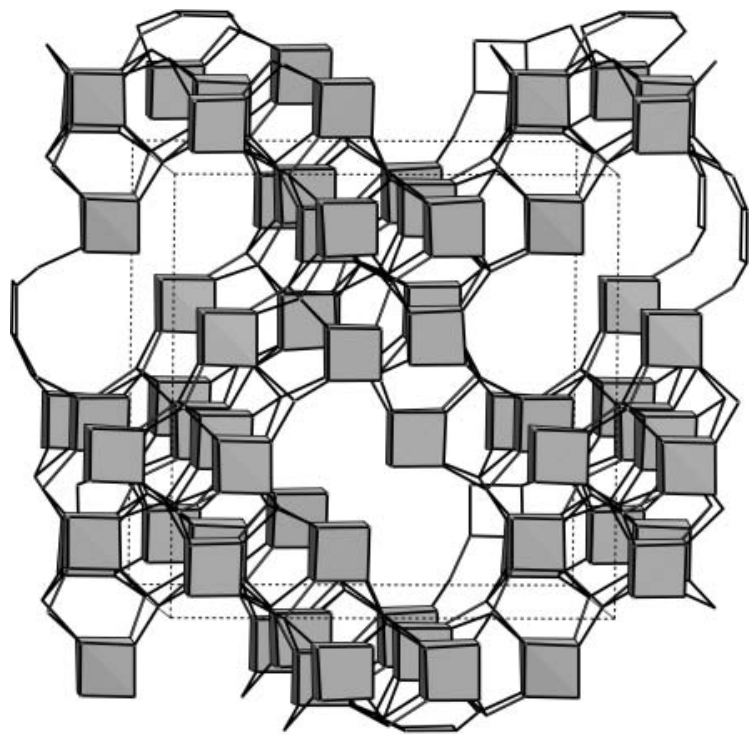

Fig. 13. The chiral framework structure of the cubic germanosilicate ITQ-37 highlighting the fluorine-centered double 4-ring building units. Nodes are $\mathrm{Ge}$ or $\mathrm{Si}$ atoms. Bridging oxygen atoms have been omitted for clarity.

intensities can also be used if sufficient care has been taken in recording them. In the case of the zeolite ITQ-26 $(\mathrm{I} / \mathrm{mmm}, \quad a=26.7769(8) \AA, \quad c=13.2505(5) \AA)$, Dorset et al. (2008) used 41 and 17 SAED intensities taken along the [001] and [100] directions, respectively [51], as input to the maximum entropy program MICE [15] to produce low resolution electrostatic potential maps. These were not sufficiently detailed to allow direct interpretation, but could be used to screen the framework structures generated by Focus from the X-ray powder diffraction data.

Sun et al. used SAED data in a somewhat different way to solve the structure of the germanosilicate zeolite ITQ-37 (P4 $132, a=26.5126(3) \AA$ A $)$ [52]. Because the unit cell is cubic and rather large, the overlap problem is quite severe. In particular, many symmetry-unrelated reflections overlap exactly in the powder pattern (e.g. 511 and 333). SAED patterns were recorded along the [100], [110], [111] and [120] directions and their intensities extracted using the program ELD [53]. To get a better estimate of the relative intensities of the reflections overlapping in the powder diffraction pattern, the ratios of the corresponding SAED intensities were used to prepartition them. This improved set of intensities was then used as input for Superflip, and the resulting electron density maps could be interpreted in a straightforward manner to produce the framework structure shown in Fig. 13.

\section{Conclusions}

The combination of X-ray powder diffraction and electron microscopy techniques is a powerful one for the determination of the structures of polycrystalline materials that resist solution by more conventional methods. Crystallographic phase information obtained from high-resolution electron microscopy images or from precession electron diffraction patterns has proven to be particularly useful. 
The powder diffraction structure determination programs Focus and $p \mathrm{CF}$, both of which operate in both direct and reciprocal space, use the phase information in the critical early stages of the structure solution procedure. Electron diffraction data, whether recorded using the conventional selected area or the newer precession technique, can also be exploited to get a better estimation of the relative intensities of overlapping reflections, and thereby a more single-crystal-like dataset. By combining data from these two different sources, several complex zeolite structures have been solved. The concepts used for these zeolite structure solutions are general, and can also be applied to other classes of materials, whose structures cannot be solved by more traditional methods.

Acknowledgements. All drawings with electron density maps or structure envelopes were generated with the Chimera visualization program [54]. The precession electron diffraction experiments were performed at the Electron Microscopy Center, ETH Zurich (EMEZ).

\section{References}

[1] W. I. F. David, K. Shankland, Structure determination from powder diffraction data, Acta Crystallogr. A 2008, 64, 52-64 and references therein.

[2] U. Kolb, E. Mugnaioli, T. E. Gorelik, Automated electron diffraction tomography - a new tool for nano crystal structure analysis, Cryst. Res. Technol. 2011, 46, 542-554.

[3] D. L. Zhang, P. Oleynikov, S. Hovmöller, X. D. Zou, Collecting 3D electron diffraction data by the rotation method, Z. Kristallogr. 2010, 225, 94-102.

[4] D. L. Dorset, Structural Electron Crystallography, Plenum Press, New York, 1995.

[5] X. D. Zou, S. Hovmöller, P. Oleynikov, Electron Crystallography, Oxford University Press, 2011.

[6] W. I. F. David, Extending the power of powder diffraction for structure determination, Nature 1990, 346, 731-734.

[7] J. Jansen, R. Peshar, H. Schenk, On the determination of accurate intensities from powder diffraction data. II. Estimation of intensities of overlapping reflections, J. Appl. Crystallogr. 1992, $25,237-243$.

[8] M. A. Estermann, V. Gramlich, Improved treatment of severely or exactly overlapping Bragg reflections for the application of direct methods to powder data, J. Appl. $\overline{\text { Crystallogr. 1993, }} 2 \overline{6}$, $396-404$.

[9] K. Shankland, W. I. F. David, D. S. Sivia, Routine ab initio structure determination of chlorothiazide by X-ray powder diffraction using optimised data collection and analysis strategies, J. Mater. Chem. 1997, 7, 569-572.

[10] M. Brunelli, J. P. Wright, G. R. M. Vaughan, A. J. Mora, A. N. Fitch, Solving larger molecular crystal structures from powder diffraction data by exploiting anisotropic thermal expansion, $A n$ gew. Chem. Int. Ed. 2003, 42, 2029-2032.

[11] Ch. Baerlocher, L. B. McCusker, eds., Structure determination from powder diffraction data, Z. Kristallogr. Special Issue 2004, 219, 782-901.

[12] U. Schmueli, Theories and Techniques of Crystal Structure Determination, Oxford University Press, 2007.

[13] A. Altomare, C. Cuocci, C. Giacovazzo, A. Moliterni, R. Rizzi, New features in EXPO2007, a program for crystal structure resolution from powder data, Croatica Chemica Acta 2008, 81, 401-407.

[14] J. Rius, C. Frontera, Application of the constrained S-FFT direct-phasing method to powder diffraction data. XIII, J. Appl. Crystallogr. 2007, 40, 1035-1038.

[15] C. Gilmore, W. Dong, G. Bricogne, A multisolution method of phase determination by combined maximization of entropy and likelihood. VI. The use of error-correcting codes as a source of phase permutation and their application to the phase problem in powder, electron and macromolecular crystallography, Acta Crystallogr. A 1999, 55, 70-83.
[16] W. I. F. David, K. Shankland, L. B. McCusker, Ch. Baerlocher, eds. Structure Determination from Powder Diffraction Data, Oxford University Press, 2002.

[17] R. Miller, G. T. DeTitta, R. Jones, D. A. Langs, C. M. Weeks, H. A. Hauptman, On the application of the minimal principle to solve unknown structures, Science 1993, 259, 1430-1433.

[18] G. Oszlányi, A. Sütô, Ab initio structure solution by charge flipping, Acta Crystallogr. A 2008, 64, 123-134.

[19] R. W. Grosse-Kunstleve, L. B. McCusker, Ch. Baerlocher, Powder diffraction data and chemical information combined in an automated structure determination procedure for zeolites J. Appl. Crystallogr. 1997, 30, 985-995.

[20] S. Brenner, L. B. McCusker, Ch. Baerlocher, Using a structure envelope to facilitate structure solution from powder diffraction data, J. Appl. Crystallogr. 1997, 30, 1167-1172.

[21] H. G. von Schnering, R. Nesper, Nodal surfaces of Fourier series: fundamental invariants of structured matter, Z. Phys. B Condensed Matter 1991, 83, 407-412.

[22] S. Brenner, L. B. McCusker, Ch. Baerlocher, The application of structure envelopes in structure determination from powder diffraction data, J. Appl. Crystallogr. 2002, 235, 243-252.

[23] R. Vincent, P. A. Midgley, Double conical beam-rocking system for measurement of integrated electron diffraction intensities, $\mathrm{Ul}$ tramicroscopy 1994, 53, 271-282

[24] P. Oleynikov, S. Hovmöller, X. D. Zou, Precession electron diffraction: observed and calculated intensities, Ultramicroscopy 2007, 107, 523-533.

[25] T. E. Weirich, J. Portillo, G. Cox, H. Hibst, S. Nicolopoulos, Ab initio determination of the framework structure of the heavy-metal oxide $\mathrm{Cs}_{\mathrm{x}} \mathrm{Nb}_{2.54} \mathrm{~W}_{2.46} \mathrm{O}_{14}$ from $100 \mathrm{kV}$ precession electron diffraction data, Ultramicroscopy 2006, 106, 164-175.

[26] D. L. Dorset, C. J. Gilmore, J. L. Jorda, S. Nicolopoulos, Direct electron crystallographic determination of zeolite zonal structures, Ultramicroscopy 2007, 107, 462-473.

[27] C. J. Gilmore, W. Dong, D. L. Dorset, Solving the crystal structures of zeolites using electron diffraction data. I. The use of potential-density histograms, Acta Crystallogr. A 2008, 64, 284-294.

[28] C. J. Gilmore, W. Dong, D. L. Dorset, Solving the crystal structures of zeolites using electron diffraction data. II. Density-building functions, Acta Crystallogr. A 2008, 64, 295-302.

[29] P. Boullay, V. Dorcet, O. Perez, C. Grygiel, W. Prellier, B. Mercey, M. Hervieu, Structure determination of a brownmillerite $\mathrm{Ca}_{2} \mathrm{Co}_{2} \mathrm{O}_{5}$ thin film by precession electron diffraction, Phys. Rev. B 2009, 79, 184108-1-184108-8.

[30] S. Hovmöller, CRISP: crystallographic image processing on a personal computer, Ultramicroscopy 1992, 41, 121-135.

[31] F. Gramm, Kombination von Transmissions-Elektronenmikroskopie mit Pulver-Beugungsdaten zur Lösung von komplexen Zeolith-Strukturen, Ph.D. Thesis, ETH Zurich, Switzerland, 2007.

[32] A. Corma, F. Rey, S. Valencia, J. L. Jorda, J. Rius, A zeolite with interconnected 8- 10- and 12-ring pores and its unique catalytic selectivity, Nature Mater. 2003, 2, 493-497.

[33] G. Oszlányi, A. Sütô, Ab initio structure solution by charge flipping, Acta Crystallogr. A 2004, 60, 134-141.

[34] G. Oszlányi, A. Sütô, Ab initio structure solution by charge flipping II. Use of weak reflections, Acta Crystallogr. A 2005, 61, $147-152$.

[35] J. Wu, K. Leinenweber, J. C. H. Spence, M. O'Keeffe, Ab initio phasing of X-ray powder diffraction patterns by charge flipping, Nature Mater. 2006, 5, 647-652.

[36] Ch. Baerlocher, L. B. McCusker, L. Palatinus, Charge flipping combined with histogram matching to solve complex crystal structures from powder diffraction data. Z. Kristallogr. 2007, 222, 47-53.

[37] L. Palatinus, G. Chapuis, SUPERFLIP - a computer program for the solution of crystal structures by charge flipping in arbitrary dimensions, J. Appl. Crystallogr. 2007, 40, 786-790.

[38] K. Y. J. Zhang, P. Main, Histogram matching as a new density modification technique for phase refinement and extension of protein molecules, Acta Crystallogr. A 1990, 46, 41-46.

[39] F. Gramm, Ch. Baerlocher, L. B. McCusker, S. J. Warrender, P. A. Wright, B. Han, S. B. Hong, Z. Liu, T. Ohsuna, O. Terasaki, Complex zeolite structure solved by combining powder diffraction and electron microscopy, Nature 2006, 444, 79-81. 
[40] Ch. Baerlocher, F. Gramm, L. Massüger, L. B. McCusker, Z. B. He, S. Hovmöller, X. D. Zou, Structure of the polycrystalline zeolite catalyst IM-5 solved by enhanced charge flipping. Science 2007, 315, 1113-1116.

[41] Ch. Baerlocher, D. Xie, L. B. McCusker, S.-J. Hwang, I. Y. Chan, K. Ong, A. W. Burton, S. I. Zones, Ordered silicon vacancies in the framework structure of the zeolite catalyst SSZ74, Nature Mater. 2008, 7, 631-635.

[42] L. B. McCusker, Ch. Baerlocher, Using electron microscopy to complement X-ray powder diffraction data to solve complex crystal structures, Chem. Commun. 2008, 1439-1451.

[43] S. B. Hong, E. G. Lear, P. A. Wright, W. Zhou, P. A. Cox, C.-H. Shin, J.-H. Park, I. S. Nam, Synthesis, structure solution, characterization, and catalytic properties of TNU-10: a high-silica zeolite with the STI topology, J. Am. Chem. Soc. 2004, 126, $5817-5826$.

[44] E. Benazzi, J. L. Guth, L. Rouleau, PCT WO 98/17581, 1998.

[45] J. L. Sun, Z. B. He, S. Hovmöller, X. D. Zou, F. Gramm, Ch. Baerlocher, L. B. McCusker, Structure determination of the zeolite IM-5 using electron crystallography alone, Z. Kristallogr. 2010, 225, 77-85.

[46] A. W. Burton, S. I. Zones, S. Elomari, I. Y. Chan, C.-Y. Chen, S.-J. Hwang, K. Ong, A comparative study of three closely related unsolved zeolite structures, Studies Surf. Sci Catal. 2007, 170, 690-697.

[47] D. Xie, Ch. Baerlocher, L. B. McCusker, Combining precession

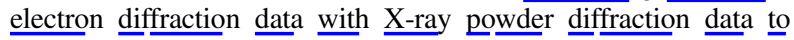

facilitate structure solution, J. Appl. Crystallogr. 2008, 41, $\overline{1115}-112 \overline{1}$

[48] D. L. Dorset, The crystal structure of ZSM-10, a powder X-ray and electron diffraction study, Z. Kristallogr. 2006, 221, 260265.

[49] D. Xie, Structure Elucidation of Polycrystalline Materials using X-ray Powder Diffraction and Electron Microscopy Techniques, Ph.D. Thesis, ETH Zurich, Switzerland, 2010.

[50] H. van Koningsveld, H. van Bekkum, J. C. Jansen, On the location and disorder of the tetrapropylammonium (TPA) ion in zeolite ZSM-5 with improved framework accuracy, Acta Crystallogr. $B$ 1987, 43, 127-132.

[51] D. L. Dorset, K. G. Strohmaier, C. E. Kliewer, A. Corma, M. J. Diaz-Cabanas, F. Rey, C. J. Gilmore, Crystal structure of ITQ26, a 3D framework with extra-large pores, Chem. Mater. 2008, 20, 5325-5331.

[52] J. L. Sun, C. Bonneau, Á Cantín, A. Corma, M. J. Díaz-Cabañas, M. Moliner, D. K, Zhang, M. R. Li, X. D. Zou, The ITQ37 mesoporous chiral zeolite, Nature 2009, 458, 1154-1158.

[53] X. D. Zou, Y. Sukharev, S. Hovmöller, Quantitative measurement of intensities from electron diffraction patterns for structure determination - new features in the program system ELD, Ultramicroscopy 1993, 52, 436-444.

[54] P. E. Pettersen, T. D. Goddard, C. C. Huang, G. S. Couch, D. M. Greenblatt, E. C. Meng, T. E. Ferrin, UCSF Chimera - a visualization system for exploratory research and analysis, J. Comput. Chem. 2004, 25, 1605-1612. 